\title{
Some results on best proximity point on star-shaped sets in probabilistic Banach (Menger) spaces
}

\author{
Hamid Shayanpour*, Maryam Shams and Asiyeh Nematizadeh
}

"Correspondence:

h.shayanpour@sci.sku.ac.ir

Department of Pure Mathematics,

University of Shahrekord,

Shahrekord, 88186-34141, Iran

\begin{abstract}
We first present the concepts of proximal contraction and proximal nonexpansive mappings on star-shaped sets in probabilistic Banach (Menger) spaces. We derive some results about the best proximity points for these mappings in probabilistic Banach (Menger) spaces. Next, we bring some examples that defend our main results. MSC: Primary 41A65; 47H10; 47H09; secondary 46S50

Keywords: proximal contraction; proximal nonexpansive mappings; best proximity point; P-property; star-shaped
\end{abstract}

\section{Introduction and preliminaries}

The equation $T x=x$ for a mapping $T: A \rightarrow B$ may have no solution whenever $A \cap B=\emptyset$, where $A, B$ are two nonempty subsets in a metric space $(X, d)$. Under this condition, it is beneficial to determine a point $a_{0} \in A$ such that $d\left(a_{0}, T a_{0}\right)$ is minimal. If $d\left(a_{0}, T a_{0}\right)$ is the global minimum value of $\operatorname{dist}(A, B)$, i.e., $d\left(a_{0}, T a_{0}\right)=\operatorname{dist}(A, B)=\min \{d(a, b): a \in A, b \in B\}$, then $a_{0}$ is called best proximity point of $T$.

In 1969, Fan [1] proved one of the most classical theorems in best approximation theory. He showed that if $(V, \rho)$ is a topological vector space with seminorm $p, W \subseteq V$, and $T$ : $W \rightarrow V$ is a mapping, then under certain conditions, there exists an element $w_{0} \in W$ such that

$$
\rho\left(w_{0}-T w_{0}\right)=d\left(T w_{0}, W\right) .
$$

Thereafter, this theorem has been generalized for continuous multivalued mappings by Reich $[2,3]$ and Sehgal and Singh [4].

Eldred et al. [5] showed that every relatively nonexpansive mapping has a proximal point under certain conditions. For further existence results of a best proximity point for several types of contractions, we refer to [6-25].

In 1942, a probabilistic metric (PM) space was introduced by Menger [26]. Schweizer and Sklar $[27,28]$ were two pioneers in the study of PM spaces.

PM spaces are very useful in probabilistic functional analysis, quantum particle physics, $\epsilon^{\infty}$ theory, nonlinear analysis, and applications; see [29-33].

Indeed, the study of fixed point results in PM spaces is one of the most active research areas in fixed point theory. Sehgal and Bharucha-Reid [34] were two pioneers in this study.

(c) 2016 Shayanpour et al. This article is distributed under the terms of the Creative Commons Attribution 4.0 International License (http://creativecommons.org/licenses/by/4.0/), which permits unrestricted use, distribution, and reproduction in any medium, provided you give appropriate credit to the original author(s) and the source, provide a link to the Creative Commons license, and indicate if changes were made. 
For further existence results of a fixed point and common fixed point in PM spaces, we refer, for example, to [35-37]. In 2014, Su and Zhang [38], proved some best proximity point theorems in PM spaces.

Let $\Delta^{+}$be the set of all distribution functions $F$ (i.e., a nondecreasing and left-continuous function $F: \mathbb{R} \rightarrow[0,1]$ such that $\inf _{t \in \mathbb{R}} F(t)=0$ and $\left.\sup _{t \in \mathbb{R}} F(t)=1\right)$ such that $F(0)=0$. Let $X$ be a nonempty set, $\epsilon_{0}=\chi_{(0, \infty)} \in \Delta^{+}$, and $F: X \times X \rightarrow \Delta^{+}\left(F(p, q)=F_{p, q}\right)$ be a mapping such that

(PM1) $F_{p, q}=\epsilon_{0}$ iff $p=q$,

(PM2) $F_{p, q}=F_{q, p}$, and

(PM3) if $F_{p, q}(t)=1$ and $F_{q, r}(s)=1$, then $F_{p, r}(t+s)=1$

for all $p, q, r \in X$ and $t, s \geq 0$. Then $(X, F)$ is called a probabilistic metric space.

For well-known definitions (such as t-norm, t-norm of $\mathrm{H}$-type, probabilistic Menger space, complete probabilistic Menger space, probabilistic normed (PN) space, etc.) and known results, we refer to $[27,39]$.

First, we state some notation, definitions, and known results; afterward, we introduce concepts of proximal contraction, proximal nonexpansive, $P$-property, weak $P$-property, and semisharp proximinal pair in PM spaces. Throughout this paper, the minimum $\mathrm{t}$-norm will be denoted by $\Delta_{m}(a, b)=\min \{a, b\}$.

Lemma 1.1 ([39]) Let $\left(x_{n}\right)$ be a sequence in a probabilistic Menger space $(X, F, \Delta)$ such that $\Delta$ is a t-norm of H-type. If

$$
F_{x_{n}, x_{n+1}}(k t) \geq F_{x_{n-1}, x_{n}}(t) \quad(n \geq 1, t>0)
$$

for some $k \in(0,1)$, then $\left(x_{n}\right)$ is a Cauchy sequence.

Definition 1.2 Suppose that $A$ is a nonempty subset of a probabilistic Menger space $(X, F, \Delta)$. Then the probabilistic diameter of $A$ is the mapping $D_{A}$ defined on $[0, \infty]$ by $D_{A}(\infty)=1$ and $D_{A}(x)=\lim _{t \rightarrow x^{-}} \varphi_{A}(t)$, where $\varphi_{A}(t)=\inf \left\{F_{a, b}(t): a, b \in A\right\}$.

A nonempty set $A$ in a probabilistic Menger space is bounded if $\lim _{x \rightarrow \infty} D_{A}(x)=1$. It is easy to see that $F_{a, b}(t) \geq D_{A}(t)$ for all $a, b \in A$ and $t \geq 0$.

Definition 1.3 Let $(X, F, \Delta)$ be a probabilistic Menger space, $A \subseteq X$, and $T: A \rightarrow A$ be a mapping. The mapping $T$ is said to be an isometry if

$$
F_{T x, T y}(t)=F_{x, y}(t) \quad \forall x, y \in X, \forall t \geq 0 .
$$

Definition 1.4 Let $(X, F, \Delta)$ be a probabilistic Menger space, and $A, B \subseteq X$. A mapping $T: A \rightarrow B$ is said to be continuous at $x \in A$ if for every sequence $\left(x_{n}\right)$ in $A$ that converges to $x$, the sequence $\left(T x_{n}\right)$ in $B$ converges to $T x$.

Remark 1.5 If $T$ is an isometry mapping on subset $A$ of a probabilistic Menger space $(X, F, \Delta)$, then $T$ is a continuous mapping because

$$
F_{T x_{n}, T x}(t)=F_{x_{n}, x}(t) \rightarrow 1 \quad \forall t>0 .
$$

Also, it is easy to see that $T$ is an injective mapping. 
An immediate consequence of the definition of a PN space ([27], Section 15.1) is the following lemma.

Lemma 1.6 ([27]) Let $(X, v, \Delta)$ be a PN space, and $F^{v}$ be the function from $X \times X$ into $\Delta^{+}$ defined by

$$
F^{v}(p, q)=v_{p-q}
$$

Then $\left(X, F^{v}, \Delta\right)$ is a probabilistic Menger space.

We call this probabilistic metric $F^{v}$ on $X$ the probabilistic metric induced by the probabilistic norm $v$.

Definition 1.7 A PN space $(X, v, \Delta)$ is said to be a probabilistic Banach space if $\left(X, F^{\nu}, \Delta\right)$ is a complete probabilistic Menger space.

Remark 1.8 Let $A, B, C$ be a nonempty subsets of a PN space $(X, v, \Delta)$ such that $\Delta$ is continuous t-norm and $x \in A$. If two mappings $T: A \rightarrow B$ and $S: A \rightarrow C$ are continuous at $x$, then $T+S$ is continuous at $x$ because

$$
v_{(T+S)(x)-(T+S)\left(x_{n}\right)}(t) \geq \Delta\left(v_{T(x)-T\left(x_{n}\right)}\left(\frac{t}{2}\right), v_{S(x)-S\left(x_{n}\right)}\left(\frac{t}{2}\right)\right) \rightarrow 1 \quad \forall t>0 .
$$

Definition 1.9 Let $A$ be a nonempty subset of a PM space (X,F). A mapping $T: A \rightarrow X$ is called a contraction (nonexpansive) if $F_{T x, T y}(t) \geq F_{x, y}\left(\frac{t}{\alpha}\right)\left(F_{T x, T y}(t) \geq F_{x, y}(t)\right)$ for some $0<$ $\alpha<1$ and for all $x, y \in A$ and $t>0$.

Definition 1.10 Suppose that $A$ and $B$ are nonempty subsets of a PM space $(X, F)$. Then the probabilistic distance of $A, B$ is the mapping $F_{A, B}$ defined on $[0, \infty]$ by

$$
F_{A, B}(t)=\sup _{x \in A, y \in B} F_{x, y}(t) \quad \forall t \geq 0
$$

Also, if $A$ and $B$ are nonempty subsets of a PN space $(X, v, \Delta)$, then $F_{A, B}^{v}(t)=v_{A-B}(t)=$ $\sup _{x \in A, y \in B} v_{x-y}(t)$, where $F^{v}$ is the probabilistic metric induced by the probabilistic norm $v$.

Definition 1.11 Let $(X, F)$ be a PM space. For subsets $A$ and $B$ of $X$, define:

$$
\begin{aligned}
& A_{0}=\left\{x \in A: \exists y \in B \text { s.t. } \forall t \geq 0, F_{x, y}(t)=F_{A, B}(t)\right\}, \\
& B_{0}=\left\{y \in B: \exists x \in A \text { s.t. } \forall t \geq 0, F_{x, y}(t)=F_{A, B}(t)\right\} .
\end{aligned}
$$

Clearly, if $A_{0}$ (or $B_{0}$ ) is a nonempty subset, then $A$ and $B$ are nonempty subsets.

Definition 1.12 Let $(X, F)$ be a PM space, and $(A, B)$ be a pair of nonempty subsets of $X$. A mapping $T: A \rightarrow B$ is called the proximal contraction (proximal nonexpansive) if there exists a real number $0<\alpha<1$ such that

$$
F_{u, T x}(t)=F_{A, B}(t)=F_{v, T y}(t) \quad \Longrightarrow \quad F_{u, v}(t) \geq F_{x, y}\left(\frac{t}{\alpha}\right)
$$




$$
\left(F_{u, T x}(t)=F_{A, B}(t)=F_{v, T y}(t) \quad \Longrightarrow \quad F_{u, v}(t) \geq F_{x, y}(t)\right)
$$

for all $u, v, x, y \in A$ and $t>0$.

Example 1.13 Let $X=[0,2]$, and $T: X \rightarrow X$ be the mapping defined by $T x=\frac{1}{8} x$. If $F_{x, y}(t)=$ $\frac{t}{t+|x-y|}$, then it is easy to check that $F_{X, X}(t)=1$. If $F_{u, T x}(t)=1=F_{v, T y}(t)$, then for $\alpha=\frac{1}{8}$, we have $F_{u, v}(t)=F_{x, y}\left(\frac{t}{\alpha}\right)$, where $u, v, x, y \in X$. Therefore, $T$ is a proximal contraction.

Definition 1.14 Let $X$ be a vector space, and $A$ be a nonempty subset of $X$. Then the subset $A$ is called a $p$-star-shaped set if there exists a point $p \in A$ such that $\alpha p+(1-\alpha) x \in A$ for all $x \in A, \alpha \in[0,1]$, and $p$ is called the center of $A$.

Clearly, each convex set $C$ is a $p$-star-shaped set for each $p \in C$. Let $\left(X, v, \Delta_{m}\right)$ be a PN space, $A$ be a $p$-star-shaped set, $B$ be a $q$-star-shaped set, and $v_{p-q}=v_{A-B}$. If $x \in A_{0}$, then there exists a point $y \in B$ such that $v_{x-y}(t)=v_{A-B}(t)$ for all $t>0$. So we have

$$
\begin{aligned}
v_{A-B}(t) & \geq v_{(\alpha p+(1-\alpha) x)-(\alpha q+(1-\alpha) y)}(t) \\
& \geq \Delta_{m}\left(v_{\alpha(p-q)}(\alpha t), v_{(1-\alpha)(x-y)}((1-\alpha) t)\right) \\
& =\Delta_{m}\left(v_{p-q}(t), v_{x-y}(t)\right) \\
& =\Delta_{m}\left(v_{A-B}(t), v_{A-B}(t)\right) \\
& =v_{A-B}(t)
\end{aligned}
$$

for all $t>0$. Therefore, $v_{(\alpha p+(1-\alpha) x)-(\alpha q+(1-\alpha) y)}(t)=v_{A-B}(t)$, which means that $A_{0}$ is a $p$-starshaped set and, similarly, that $B_{0}$ is a $q$-star-shaped set.

Definition 1.15 Let $(X, F)$ be a PM space. A pair $(A, B)$ of nonempty subsets of $X$ is said to have the $P$-property (weak $P$-property) if $A_{0} \neq \emptyset$ and

$$
\begin{aligned}
& F_{u, x}(t)=F_{A, B}(t)=F_{v, y}(t) \quad \Longrightarrow \quad F_{u, v}(t)=F_{x, y}(t) \\
& \left(F_{u, x}(t)=F_{A, B}(t)=F_{v, y}(t) \quad \Longrightarrow \quad F_{u, v}(t) \geq F_{x, y}(t)\right)
\end{aligned}
$$

for all $u, v \in A_{0}, x, y \in B_{0}$, and $t>0$.

Example 1.16 Let $X=\mathbb{R}^{2}$ and define

$$
F_{(x, y),(u, v)}(t)=\frac{t}{t+\sqrt{(x-u)^{2}+(y-v)^{2}}} .
$$

Clearly, $\left(X, F, \Delta_{m}\right)$ is a complete probabilistic Menger space. Let

$$
\begin{aligned}
& A=\left\{\left(0, \frac{1}{n}\right): n \in \mathbb{N}\right\} \cup\{(0,0)\}, \\
& B=\left\{\left(1, \frac{1}{n}\right): n \in \mathbb{N}\right\} \cup\{(1,0)\} .
\end{aligned}
$$


Then it is easy to check that $A_{0}=A, B_{0}=B$, and $F_{A, B}(t)=\frac{t}{t+1}$. If

$$
F_{(0, x),(1, y)}(t)=F_{A, B}(t)=\frac{t}{t+1}=F_{(0, u),(1, v)}(t),
$$

then $x=y$ and $u=v$, so that

$$
F_{(0, x),(0, u)}(t)=\frac{t}{t+|x-u|}=\frac{t}{t+|y-v|}=F_{(1, y),(1, v)}(t) .
$$

Therefore, the pair $(A, B)$ has the $P$-property.

Example 1.17 Let $X=\mathbb{R}^{2}$ and define

$$
F_{(x, y),(u, v)}(t)=\frac{t}{t+\sqrt{(x-u)^{2}+(y-v)^{2}}} .
$$

Let $A=\{(0,0)\}$ and $B=\left\{(x, y) \in X: y=1+\sqrt{1-x^{2}}\right\}$. Clearly, $A_{0}=\{(0,0)\}$ and $B_{0}=$ $\{(-1,1),(1,1)\}$. If

$$
F_{(0,0),(x, y)}(t)=F_{A, B}(t)=\frac{t}{t+\sqrt{2}}=F_{(0,0),(u, v)}(t),
$$

then

$$
1=F_{(0,0),(0,0)}(t) \geq F_{(x, y),(u, v)}(t),
$$

where $(x, y),(u, v) \in B_{0}$. Therefore, the pair $(A, B)$ has the weak $P$-property.

Definition 1.18 Let $(X, F)$ be a PM space. A pair $(A, B)$ of nonempty subsets of $X$ is called a semisharp proximinal pair if there exists at most one $\left(x_{0}, y_{0}\right) \in A \times B$ such that $F_{x, y_{0}}(t)=$ $F_{A, B}(t)=F_{x_{0}, y}(t)$ for all $(x, y) \in A \times B$.

It is easy to check that if a pair $(A, B)$ has the $P$-property, then the pair $(A, B)$ is a semisharp proximinal pair. Clearly, a semisharp proximinal pair $(A, B)$ does not necessarily have the $P$-property.

Example 1.19 Suppose that $X=\mathbb{R}, A=\{-10,10\}, B=\{-2,2\}$, and $F_{x, y}(t)=\frac{t}{t+|x-y|}$. It is easy to verify that $F_{A, B}(t)=\frac{t}{t+8}, A_{0}=A, B_{0}=B$, and $(A, B)$ is a semisharp proximinal pair but does not have the $P$-property.

Remark 1.20 It is easy to check that the $P$-property is stronger than the weak $P$-property. If a pair $(A, B)$ has the weak $P$-property and $T: A \rightarrow B$ is a nonexpansive mapping, then for all $u, v, x, y \in A$, we have

$$
F_{u, T x}(t)=F_{A, B}(t)=F_{v, T y}(t) \quad \Longrightarrow \quad F_{u, v}(t) \geq F_{T x, T y}(t) \geq F_{x, y}(t) .
$$

That is, $T$ is a proximal nonexpansive mapping. Similarly, if a pair $(A, B)$ has the weak $P$-property and $T: A \rightarrow B$ is a contraction mapping, then $T$ is a proximal contraction mapping. Also, a pair $(A, B)$ has the $P$-property if and only if both pairs $(A, B)$ and $(B, A)$ have the weak $P$-property. 
Definition 1.21 Let $X$ and $Y$ be vector spaces. A mapping $T: X \rightarrow Y$ is affine if

$$
T\left(\sum_{i=1}^{n} \lambda_{i} x_{i}\right)=\sum_{i=1}^{n} \lambda_{i} T\left(x_{i}\right)
$$

for all $n \in \mathbb{N}, x_{1}, \ldots, x_{n} \in X$, and $\lambda_{1}, \ldots, \lambda_{n} \in \mathbb{R}$ such that $\sum_{i=1}^{n} \lambda_{i}=1$.

In Section 2, we show some results on the best proximity points in probabilistic Banach (Menger) spaces. For example, if $(A, B)$ is a semisharp proximinal pair of a probabilistic Banach space $\left(X, v, \Delta_{m}\right)$ such that $A$ is a $p$-star-shaped set, $A_{0}$ is a nonempty compact set, $B$ is a $q$-star-shaped set and $v_{p-q}(t)=v_{A-B}(t)$ for all $t>0$, then every proximal nonexpansive mapping $T: A \rightarrow B$ with $T\left(A_{0}\right) \subseteq B_{0}$ has a best proximity point. We also prove that if $A$ is a nonempty, compact, and convex subset of a probabilistic Banach space $\left(X, v, \Delta_{m}\right)$ and $T: A \rightarrow A$ is a nonexpansive mapping, then $T$ has a fixed point. Finally, we give some examples which defend our main results.

\section{Proximity point for proximal contraction and proximal nonexpansive mappings}

We first give the following lemma and then we state the main results of this paper. We recall that if $A_{0}$ (or $B_{0}$ ) is a nonempty subset, then $A$ and $B$ are nonempty subsets.

Lemma 2.1 Let $(X, F, \Delta)$ be a complete probabilistic Menger space such that $\Delta$ is a $t$-norm of H-type, and $A, B \subseteq X$ be such that $A_{0}$ is a nonempty closed set. If $T: A \rightarrow B$ is a proximal contraction mapping such that $T\left(A_{0}\right) \subseteq B_{0}$, then there exists a unique $x \in A_{0}$ such that $F_{x, T x}(t)=F_{A, B}(t)$ for all $t>0$.

Proof Since $A_{0}$ is nonempty and $T\left(A_{0}\right) \subseteq B_{0}$, there exist $x_{1}, x_{0} \in A_{0}$ such that $F_{x_{1}, T x_{0}}(t)=$ $F_{A, B}(t)$. Since $T x_{1} \in B_{0}$, there exists $x_{2} \in A_{0}$ such that $F_{x_{2}, T x_{1}}(t)=F_{A, B}(t)$. Continuing this process, we obtain a sequence $\left(x_{n}\right) \subseteq A_{0}$ such that $F_{x_{n+1}, T x_{n}}(t)=F_{A, B}(t)$ for all $n \in \mathbb{N}$ and $t>0$. Since for all $n \in \mathbb{N}$,

$$
F_{x_{n}, T x_{n-1}}(t)=F_{A, B}(t)=F_{x_{n+1}, T x_{n}}(t) \quad(t>0)
$$

and $T$ is a proximal contraction, we have

$$
F_{x_{n+1}, x_{n}}(t) \geq F_{x_{n}, x_{n-1}}\left(\frac{t}{\alpha}\right) \quad(0<\alpha<1, t>0) .
$$

Therefore, by Lemma 1.1, $\left(x_{n}\right)$ is a Cauchy sequence and so converges to some $x \in A_{0}$. Again by the assumption $T\left(A_{0}\right) \subseteq B_{0}, T x \in B_{0}$. Then there exists an element $u \in A_{0}$ such that $F_{u, T x}(t)=F_{A, B}(t)$ for all $t>0$. Since for all $n \in \mathbb{N}$,

$$
F_{u, T x}(t)=F_{A, B}(t)=F_{x_{n+1}, T x_{n}}(t) \quad(t>0),
$$

by the hypothesis we have

$$
F_{u, x_{n+1}}(t) \geq F_{x, x_{n}}\left(\frac{t}{\alpha}\right) \geq F_{x, x_{n}}(t) \quad(t>0) .
$$


Letting $n \rightarrow \infty$ shows that $x_{n} \rightarrow u$ and thus $x=u$, so $F_{x, T x}(t)=F_{A, B}(t)$. If there exists another element $y$ such that $F_{y, T y}(t)=F_{A, B}(t)$, then by the hypothesis we have $F_{x, y}(t) \geq F_{x, y}\left(\frac{t}{\alpha}\right)$, which means that $x=y$.

Proposition 2.2 Let $(X, F, \Delta)$ be a probabilistic Menger space, and $A, B \subseteq X$ be such that $A_{0}$ is a nonempty set. Suppose that $T: A \rightarrow B$ is a proximal contraction mapping such that $T\left(A_{0}\right) \subseteq B_{0}$ and $g: A \rightarrow A$ is an isometry mapping such that $A_{0} \subseteq g\left(A_{0}\right)$. Denote $G=g(A)$ and

$$
G_{0}=\left\{z \in G: \exists y \in B \text { s.t. } \forall t \geq 0, F_{z, y}(t)=F_{G, B}(t)\right\} .
$$

Then $\mathrm{Tg}^{-1}$ is a proximal contraction, and $G_{0}=A_{0}$.

Proof Since $G \subseteq A, F_{G, B}(t) \leq F_{A, B}(t)$ for all $t>0$. Assume that $x \in A_{0} \subseteq g\left(A_{0}\right)$. Then $x=$ $g\left(x^{\prime}\right)$ for some $x^{\prime} \in A_{0}$, and so there exists $y \in B$ such that $F_{A, B}(t)=F_{g\left(x^{\prime}\right), y}(t) \leq F_{G, B}(t)$ for all $t>0$. Thus, $F_{A, B}(t)=F_{G, B}(t)$ for all $t>0$. Now we show that $\mathrm{Tg}^{-1}$ is a proximal contraction. To this end, suppose that $u, v, x, y \in G$ are such that

$$
F_{u, T g^{-1} x}(t)=F_{G, B}(t)=F_{A, B}(t)=F_{v, T g^{-1} y}(t) \quad(t>0) .
$$

By the hypothesis we have

$$
F_{u, \nu}(t) \geq F_{g^{-1} x, g^{-1} y}\left(\frac{t}{\alpha}\right)=F_{g g^{-1} x, g g^{-1} y}\left(\frac{t}{\alpha}\right)=F_{x, y}\left(\frac{t}{\alpha}\right) \quad(t>0)
$$

for some $\alpha \in(0,1)$. Therefore, $\mathrm{Tg}^{-1}$ is a proximal contraction. If $x \in G_{0}$, then $x \in G \subseteq A$, and there exists $y \in B$ such that $F_{x, y}(t)=F_{G, B}(t)=F_{A, B}(t)$ for all $t>0$, so that $x \in A_{0}$. If $x \in A_{0} \subseteq A$, then there exists $y \in B$ such that $F_{x, y}(t)=F_{A, B}(t)=F_{G, B}(t)$ for all $t>0$. On the other hand, by the hypothesis $x \in G$, and therefore $G_{0}=A_{0}$.

Corollary 2.3 Let the hypotheses of Lemma 2.1 be satisfied. Suppose that $T: A \rightarrow B$ is a proximal contraction mapping such that $T\left(A_{0}\right) \subseteq B_{0}$ and $g: A \rightarrow A$ is an isometry mapping such that $A_{0} \subseteq g\left(A_{0}\right)$. Then there exists a unique $x \in A_{0}$ such that $F_{g x, T x}(t)=F_{A, B}(t)$.

Proof By Proposition 2.2, $\mathrm{Tg}^{-1}: G=g(A) \rightarrow B$ is proximal contraction, and $\mathrm{Tg}^{-1}\left(G_{0}\right)=$ $T^{-1}\left(A_{0}\right) \subseteq T\left(A_{0}\right) \subseteq B_{0}$. Now by Lemma 2.1 there exists a unique $x^{\prime} \in A_{0}$ such that $F_{x^{\prime}, T g^{-1} x^{\prime}}(t)=F_{A, B}(t)$. Since $A_{0} \subseteq g\left(A_{0}\right)$, there exists $x \in A_{0}$ such that $x^{\prime}=g(x)$, so that $F_{g(x), T x}(t)=F_{A, B}(t)$. Note that $g$ is an injective mapping, therefore, by Lemma 2.1, $x$ is unique, and hence the result follows.

Theorem 2.4 Let $\left(X, v, \Delta_{m}\right)$ be a probabilistic Banach space, $A, B \subseteq X$ be such that $A$ is a convex set, $A_{0}$ be a nonempty compact set, and $B$ be a bounded convex set. Suppose that $T$ : $A \rightarrow B$ is a continuous affine and proximal nonexpansive mapping such that $T\left(A_{0}\right) \subseteq B_{0}$ and $g: A \rightarrow A$ is an isometry mapping such that $A_{0} \subseteq g\left(A_{0}\right)$. Then there exists an element $x \in A_{0}$ such that $v_{g x-T x}(t)=v_{A-B}(t)$ for all $t>0$.

Proof Fix $z \in A_{0}$ and $i \in(0,1)$. We define the mapping $T_{i}: A \rightarrow B$ by

$$
T_{i} x=(1-i) T z+i T x
$$


We show that $T_{i}$ is a proximal contraction. Let $u, v, x, y \in A$ be such that

$$
v_{u-T_{i} x}(t)=v_{A-B}(t)=v_{v-T_{i} y}(t) \quad(t>0) .
$$

Since $T$ is an affine mapping, we have

$$
v_{u-T((1-i) z+i x)}(t)=v_{A-B}(t)=v_{v-T((1-i) z+i y)}(t) \quad(t>0) .
$$

So by the hypothesis we have

$$
\begin{aligned}
v_{u-v}(t) & \geq v_{(1-i) z+i x-(1-i) z-i y}(t) \\
& =v_{i(x-y)}(t)=v_{x-y}\left(\frac{t}{i}\right) \quad(t>0) .
\end{aligned}
$$

Hence, $T_{i}$ is a proximal contraction. Let $x \in A_{0}$, so that $T x \in B_{0}$ and $T z \in B_{0}$. Therefore, there exist $u, v \in A_{0}$ such that

$$
v_{u-T x}(t)=v_{A-B}(t)=v_{v-T z}(t) \quad(t>0)
$$

Put $y=i u+(1-i) v \in A$. Then

$$
\begin{aligned}
v_{y-T_{i} x}(t) & =v_{i u+(1-i) v-(1-i) T z-i T x}(t) \\
& =v_{i(u-T x)+(1-i)(v-T z)}(t) \\
& \geq \Delta_{m}\left(v_{i(u-T x)}(i t), v_{(1-i)(v-T z)}((1-i) t)\right) \\
& =\Delta_{m}\left(v_{u-T x}(t), v_{v-T z}(t)\right) \\
& =\Delta_{m}\left(v_{A-B}(t), v_{A-B}(t)\right)=v_{A-B}(t) \quad(t>0),
\end{aligned}
$$

and thus $T_{i}\left(A_{0}\right) \subseteq B_{0}$. By Corollary 2.3 there exists a unique $x_{i} \in A_{0}$ such that $v_{g x_{i}-T_{i} x_{i}}(t)=$ $v_{A-B}(t)$ for all $t>0$. Fix $j \in(0,1)$. Then

$$
\begin{aligned}
v_{g x_{i}-T x_{i}}(t) & \geq \Delta_{m}\left(v_{g x_{i}-T_{i} x_{i}}(j t), v_{T_{i} x_{i}-T x_{i}}((1-j) t)\right) \\
& =\Delta_{m}\left(v_{A-B}(j t), v_{(1-i)\left(T z-T x_{i}\right)}((1-j) t)\right) \\
& =\Delta_{m}\left(v_{A-B}(j t), v_{T z-T x_{i}}\left(\frac{(1-j) t}{1-i}\right)\right) \\
& \geq \Delta_{m}\left(v_{A-B}(j t), D_{B}\left(\frac{(1-j) t}{1-i}\right)\right) \quad(t>0) .
\end{aligned}
$$

Now letting $i \rightarrow 1$, we obtain

$$
\lim _{i \rightarrow 1} v_{g x_{i}-T x_{i}}(t) \geq \Delta_{m}\left(v_{A-B}(j t), 1\right)=v_{A-B}(j t) \quad(\forall j \in(0,1), t>0) .
$$

Then letting $j \rightarrow 1$, we have

$$
\lim _{i \rightarrow 1} v_{g x_{i}-T x_{i}}(t)=v_{A-B}(t) \quad(t>0) .
$$


So we can create a sequence $\left(x_{n}\right)$ in $A_{0}$ such that

$$
v_{g x_{n}-T x_{n}}(t) \rightarrow v_{A-B}(t) \quad(t>0)
$$

Since $A_{0}$ is compact, the sequence $\left(x_{n}\right)$ has a subsequence $\left(x_{n_{k}}\right)$ such that $x_{n_{k}} \rightarrow x \in A_{0}$. By Remark $1.5, g$ is continuous mapping, and so $g-T$ is a continuous mapping by Remark 1.8 . Indeed, since $\Delta_{m}$ is a continuous t-norm, $p \rightarrow v_{P}$ is continuous ([27], Chapter 12), and we get

$$
v_{g x-T x}(t)=\lim _{k \rightarrow \infty} v_{g x_{n_{k}}-T x_{n_{k}}}(t)=v_{A-B}(t),
$$

as required.

Theorem 2.5 Let $(X, F, \Delta)$ be a complete probabilistic Menger space such that $\Delta$ is a $t$-norm of $H$-type, and $(A, B)$ be a pair of subsets of $X$ with the weak P-property such that $A_{0}$ is a nonempty closed set. If $T: A \rightarrow B$ is a contraction mapping such that $T\left(A_{0}\right) \subseteq B_{0}$, then there exists a unique $x$ in $A$ such that $F_{x, T x}(t)=F_{A, B}(t)$ for all $t>0$.

Proof It is a direct consequence of Remark 1.20 and Lemma 2.1.

Clearly, the pair $(A, A)$ has the $P$-property, so we have the following result.

Corollary 2.6 Let $(X, F, \Delta)$ be a complete probabilistic Menger space such that $\Delta$ is a t-norm of H-type. Then every contraction self-mapping from each nonempty closed subset of $X$ has a unique fixed point.

Theorem 2.7 Let $\left(X, v, \Delta_{m}\right)$ be a probabilistic Banach space, and $(A, B)$ be a semisharp proximinal pair of $X$ such that $A$ is a p-star-shaped set, $A_{0}$ be a nonempty compact set, $B$ be a q-star-shaped set, and let $v_{p-q}(t)=v_{A-B}(t)$ for all $t>0$. If $T: A \rightarrow B$ is a proximal nonexpansive mapping such that $T\left(A_{0}\right) \subseteq B_{0}$, then there exists an element $x \in A_{0}$ such that $v_{x-T x}(t)=v_{A-B}(t)$ for all $t>0$.

Proof For each integer $i \geq 1$, define $T_{i}: A_{0} \rightarrow B_{0}$ by

$$
T_{i}(x)=\left(1-\frac{1}{i}\right) T x+\frac{1}{i} q \quad\left(x \in A_{0}\right)
$$

Then by the hypothesis we have $T_{i}\left(A_{0}\right) \subseteq B_{0}$. Next, we show that for each $i, T_{i}$ is a proximal contraction with $\alpha=1-\frac{1}{i}<1$. To do this, suppose that $x, y, u, v, s, r \in A_{0}$ and $t>0$ are such that

$$
v_{u-T_{i} x}(t)=v_{v-T_{i} y}(t)=v_{A_{0}-B_{0}}(t)=v_{A-B}(t)=v_{s-T x}(t)=v_{r-T y}(t)
$$

Now we define

$$
u^{\prime}=\left(1-\frac{1}{i}\right) s+\frac{1}{i} p \in A_{0}, \quad v^{\prime}=\left(1-\frac{1}{i}\right) r+\frac{1}{i} p \in A_{0}
$$


so we have

$$
\begin{aligned}
v_{A-B}(t) & \geq v_{u^{\prime}-T_{i} x}(t)=v_{\left(1-\frac{1}{i}\right) s+\frac{1}{i} p-\left(1-\frac{1}{i}\right) T x-\frac{1}{i} q}(t) \\
& =v_{\left(1-\frac{1}{i}\right)(s-T x)+\frac{1}{i}(p-q)}(t) \\
& \geq \Delta_{m}\left(v_{\left(1-\frac{1}{i}\right)(s-T x)}\left(t\left(1-\frac{1}{i}\right)\right), v_{\frac{1}{i}(p-q)}\left(t\left(\frac{1}{i}\right)\right)\right) \\
& =\Delta_{m}\left(v_{s-T x}(t), v_{p-q}(t)\right) \\
& =\Delta_{m}\left(v_{A-B}(t), v_{A-B}(t)\right)=v_{A-B}(t) .
\end{aligned}
$$

Hence, $v_{u^{\prime}-T_{i} x}(t)=v_{A-B}(t)$. Since $v_{u-T_{i} x}(t)=v_{A-B}(t)$ and $(A, B)$ is a semisharp proximinal pair, we have $u^{\prime}=u$. By the same method we also have $v^{\prime}=v$. Since $T$ is a proximal nonexpansive mapping, we have

$$
\begin{aligned}
v_{u-v}(t) & =v_{u^{\prime}-v^{\prime}}(t)=v_{\left(1-\frac{1}{i}\right)(s-r)}(t) \\
& =v_{s-r}\left(\frac{t}{1-\frac{1}{i}}\right) \geq v_{x-y}\left(\frac{t}{1-\frac{1}{i}}\right) .
\end{aligned}
$$

Therefore, $T_{i}$ is a proximal contraction with $\alpha=1-\frac{1}{i}<1$. By Lemma 2.1, for each $i \geq 1$, there exists a unique $u_{i} \in A_{0}$ such that $v_{u_{i}-T_{i} u_{i}}(t)=v_{A_{0}-B_{0}}(t)=v_{A-B}(t)$. Since $A_{0}$ is compact and $\left(u_{i}\right) \subseteq A_{0}$, without loss of generality, we can assume that $u_{i}$ is a convergent sequence and $u_{i} \rightarrow x \in A_{0}$.

For each $i \geq 1$, since $T\left(u_{i}\right) \in T\left(A_{0}\right) \subseteq B_{0}$, there exists $v_{i} \in A_{0}$ such that $v_{v_{i}-T u_{i}}(t)=v_{A-B}(t)$. So we have

$$
\begin{aligned}
v_{A-B}(t) & \geq v_{\left(1-\frac{1}{i}\right) v_{i}+\frac{1}{i} p-T_{i} u_{i}}(t) \\
& =v_{\left(1-\frac{1}{i}\right) v_{i}+\frac{1}{i} p-\left(1-\frac{1}{i}\right) T u_{i}-\frac{1}{i} q}(t) \\
& \geq \Delta_{m}\left(v_{\left(1-\frac{1}{i}\right)\left(v_{i}-T u_{i}\right)}\left(t\left(1-\frac{1}{i}\right)\right), v_{\frac{1}{i}(p-q)}\left(t\left(\frac{1}{i}\right)\right)\right) \\
& =\Delta_{m}\left(v_{v_{i}-T u_{i}}(t), v_{p-q}(t)\right) \\
& =\Delta_{m}\left(v_{A-B}(t), v_{A-B}(t)\right)=v_{A-B}(t) .
\end{aligned}
$$

Thus, $v_{A-B}(t)=v_{\left(1-\frac{1}{i}\right) v_{i}+\frac{1}{i} p-T_{i} u_{i}}(t)$. Since $(A, B)$ is a semisharp proximinal pair and $v_{A-B}(t)=$ $v_{u_{i}-T_{i} u_{i}}(t)$, we have $u_{i}=\left(1-\frac{1}{i}\right) v_{i}+\frac{1}{i} p$, and so

$$
v_{u_{i}-v_{i}}(t)=v_{\frac{1}{i}\left(v_{i}-p\right)}(t)=v_{v_{i}-p}(i t) .
$$

Since $A_{0}$ is compact and $\left(v_{i}\right) \subseteq A_{0}$, without loss of generality, we can assume that $v_{i}$ is a convergent sequence and $v_{i} \rightarrow z \in A_{0}$. For every $j \leq i$, we have

$$
v_{u_{i}-v_{i}}(t)=v_{v_{i}-p}(i t) \geq v_{v_{i}-p}(j t) \geq \Delta_{m}\left(v_{v_{i}-z}\left(\frac{j}{2} t\right), v_{z-p}\left(\frac{j}{2} t\right)\right) .
$$


Letting $i \rightarrow \infty$, we have

$$
\lim _{i \rightarrow \infty} v_{u_{i}-v_{i}}(t) \geq v_{z-p}\left(\frac{j}{2} t\right) \quad(\forall j \geq 1) .
$$

Now letting $j \rightarrow \infty$, we have

$$
\lim _{i \rightarrow \infty} v_{u_{i}-v_{i}}(t) \geq \lim _{j \rightarrow \infty} v_{z-p}\left(\frac{j}{2} t\right)=1
$$

Therefore, $v_{u_{i}-v_{i}}(t) \rightarrow 1$, so that $z=\lim _{i \rightarrow \infty} v_{i}=\lim _{i \rightarrow \infty} u_{i}=x$. Since $T x \in B_{0}$, there must exist $u \in A_{0}$ such that $v_{A-B}(t)=v_{u-T x}(t)$. Since we know that $v_{A-B}(t)=v_{v_{i}-T u_{i}}(t)$ and $T$ is a proximal nonexpansive mapping, it follows that $v_{v_{i}-u}(t) \geq v_{u_{i}-x}(t) \rightarrow 1$. This implies that $u=\lim _{i \rightarrow \infty} v_{i}=x$ and then $v_{A-B}(t)=v_{x-T x}(t)$, as required.

Theorem 2.8 Let $\left(X, v, \Delta_{m}\right)$ be a probabilistic Banach space, $(A, B)$ be a semisharp proximinal pair of $X$ with the weak P-property such that $A$ is a p-star-shaped set, $A_{0}$ be a nonempty compact set, $B$ be a q-star-shaped set, and let $v_{p-q}(t)=v_{A-B}(t)$ for all $t>0$. If $T: A \rightarrow B$ is a nonexpansive mapping such that $T\left(A_{0}\right) \subseteq B_{0}$, then $T$ has a best proximity point in $A_{0}$.

Proof It is a direct consequence of Remark 1.20 and Theorem 2.7.

Proposition 2.9 Let $(X, F, \Delta)$ be a probabilistic Menger space, and $A, B \subseteq X$ be such that $A_{0}$ is a nonempty set. Suppose that $T: A \rightarrow B$ is a proximal nonexpansive mapping such that $T\left(A_{0}\right) \subseteq B_{0}$ and $g: A \rightarrow A$ is an isometry mapping such that $A_{0} \subseteq g\left(A_{0}\right)$. Denote $G=g(A)$ and

$$
G_{0}=\left\{z \in G: \exists y \in B \text { s.t. } \forall t \geq 0, F_{z, y}(t)=F_{G, B}(t)\right\} .
$$

Then $\mathrm{Tg}^{-1}$ is a proximal nonexpansive, and $G_{0}=A_{0}$.

Proof The result follows by using a similar argument as in the proof of Proposition 2.2.

The following theorem is an immediate consequence of Theorem 2.7 and Proposition 2.9.

Theorem 2.10 Let $\left(X, v, \Delta_{m}\right)$ be a probabilistic Banach space, $(A, B)$ be a semisharp proximinal pair of $X$ such that $A$ is a $p$-star-shaped set, $A_{0}$ be a nonempty compact set, $B$ be a $q$-star-shaped set, and let $v_{p-q}(t)=v_{A-B}(t)$ for all $t>0$. If $T: A \rightarrow B$ is a proximal nonexpansive mapping such that $T\left(A_{0}\right) \subseteq B_{0}$ and $g: A \rightarrow A$ is an isometry mapping such that $A_{0} \subseteq g\left(A_{0}\right)$, then there exists an element $x \in A_{0}$ such that $v_{g x-T x}(t)=v_{A-B}(t)$ for all $t>0$.

Corollary 2.11 Let $\left(X, v, \Delta_{m}\right)$ be a probabilistic Banach space, and let $(A, B)$ be a pair of convex subsets of $X$ with the P-property such that $A_{0}$ is a nonempty compact set. If $T: A \rightarrow$ $B$ is a nonexpansive mapping such that $T\left(A_{0}\right) \subseteq B_{0}$ and $g: A \rightarrow A$ is an isometry mapping such that $A_{0} \subseteq g\left(A_{0}\right)$, then there exists an element $x \in A_{0}$ such that $v_{g x-T x}(t)=v_{A-B}(t)$ for all $t>0$. 
In Corollary 2.11, if $g(x)=x$, then we have the following corollary.

Corollary 2.12 With the hypotheses of the previous corollary, if $T: A \rightarrow B$ is a nonexpansive mapping such that $T\left(A_{0}\right) \subseteq B_{0}$, then $T$ has a best proximity point.

In Corollary 2.12, if $A=B$, then we have the following corollary.

Corollary 2.13 If $A$ is a nonempty, compact, and convex subset of a probabilistic Banach space $\left(X, v, \Delta_{m}\right)$ and $T: A \rightarrow A$ is a nonexpansive mapping, then $T$ has a fixed point.

In the following, we give some examples that defend our main results.

Example 2.14 Let $X=\mathbb{R}^{2}, A=\{(0, y): y \in \mathbb{R}\}$ and $B=\{(1, y): y \in \mathbb{R}\}$. Suppose that $T: A \rightarrow B$ is defined by $T(0, y)=\left(1, \frac{y}{4}\right), g: A \rightarrow A$ is defined by $g(0, y)=(0,-y)$, and $F_{\left(x, x^{\prime}\right),\left(y, y^{\prime}\right)}(t)=\frac{t}{t+|x-y|+\left|x^{\prime}-y^{\prime}\right|}$. It is easy to see that $\left(X, F, \Delta_{m}\right)$ is a complete probabilistic Menger space, $F_{A, B}(t)=\frac{t}{t+1}, A_{0}=A, B_{0}=B, T\left(A_{0}\right) \subseteq B_{0}$, and

$$
F_{g(0, x), g(0, y)}(t)=F_{(0,-x),(0,-y)}(t)=\frac{t}{t+|x-y|}=F_{(0, x),(0, y)}(t) .
$$

If $(0, u),(0, x),(0, v),(0, y) \in A$ are such that

$$
\frac{t}{t+1+\left|u-\frac{x}{4}\right|}=F_{(0, u), T(0, x)}(t)=F_{A, B}(t)=F_{(0, v), T(0, y)}(t)=\frac{t}{t+1+\left|v-\frac{y}{4}\right|},
$$

then $u=\frac{x}{4}$ and $v=\frac{y}{4}$, so that

$$
F_{(0, u),(0, v)}(t)=F_{\left(0, \frac{x}{4}\right),\left(0, \frac{y}{4}\right)}(t)=\frac{t}{t+\frac{1}{4}|x-y|}=F_{(0, x),(0, y)}\left(\frac{t}{\frac{1}{4}}\right) .
$$

Therefore, all the hypothesis of Corollary 2.3 are satisfied, and we also have

$$
F_{(0,0), T(0,0)}(t)=F_{(0,0),(1,0)}(t)=\frac{t}{t+1}=F_{A, B}(t)
$$

Example 2.15 Let $X=\mathbb{R}, A=[0,2]$ and $B=[3,5]$. For every $x \in X$, define $v_{x}(t)=\frac{t}{t+|x|}$. It is easy to see that $\left(X, v, \Delta_{m}\right)$ is a probabilistic Banach space, $v_{A-B}(t)=\frac{t}{t+1}, A_{0}=\{2\}$, and $B_{0}=\{3\}$. For every $x \in A$, define $T: A \rightarrow B$ by $T x=5-x$ and let $g$ be the identity mapping. Clearly, $T$ is a continuous affine and proximal nonexpansive mapping, and $T\left(A_{0}\right)=\{T(2)\}=\{3\}=B_{0}$. Therefore, all the hypotheses of Theorem 2.4 are satisfied, and also we have

$$
v_{2-T 2}(t)=v_{2-3}(t)=\frac{t}{t+1}=v_{A-B}(t) .
$$

The following example shows that the weak $P$-property of the pair $(A, B)$ cannot be removed from Theorem 2.5 .

Example 2.16 Let $X=\mathbb{R}, A=\{-10,10\}, B=\{-2,2\}$, and $F_{p, q}(t)=\frac{t}{t+|p-q|}$. Clearly, $\left(X, F, \Delta_{m}\right)$ is a complete probabilistic Menger space. Then $A_{0}=A, B_{0}=B$, and $F_{A, B}(t)=\frac{t}{t+8}$. Let $T$ : 
$A \rightarrow B$ be a mapping given by $T(-10)=2$ and $T(10)=-2$. It is easy to see that for $\alpha=\frac{1}{5}, T$ is a contraction mapping with $T\left(A_{0}\right) \subseteq B_{0}$. The mapping $T$ does not have any best proximity point because $F_{x, T x}(t)=\frac{t}{t+12}<\frac{t}{t+8}=F_{A, B}(t)$ for all $x \in A$. It should be noted that the pair $(A, B)$ does not have the weak $P$-property.

Example 2.17 Let $X=\mathbb{R}, A=[0,1]$, and $B=[2,3]$. For every $x \in X$, define $v_{x}(t)=\frac{t}{t+|x|}$. It is easy to see that $\left(X, v, \Delta_{m}\right)$ is a probabilistic Banach space, $A$ is 1-star-shaped set, $B$ is 2-star-shaped set,

$$
v_{A-B}(t)=\sup _{x \in A, y \in B} v_{x-y}(t)=\frac{t}{t+1}, \quad A_{0}=\{1\}, \quad B_{0}=\{2\},
$$

and

$$
v_{1-2}(t)=\frac{t}{t+|1-2|}=\frac{t}{t+1}=v_{A-B}(t)
$$

Also, $(A, B)$ is a semisharp proximinal pair. Now for each $x \in A$, define $T: A \rightarrow B$ by $T x=$ $3-x$. If $u, v, x, y \in A$, then

$$
\frac{t}{t+|u-3+x|}=v_{u-T x}(t)=v_{A-B}(t)=v_{v-T y}(t)=\frac{t}{t+|v-3+y|},
$$

so that $u=x=1$ and $v=y=1$. Thus,

$$
v_{u-v}(t)=1=v_{x-y}(t)
$$

So $T$ is a proximal nonexpansive, and $T\left(A_{0}\right)=B_{0}$. Therefore, all the hypotheses of Theorem 2.7 are satisfied, and we also have

$$
v_{1-T 1}(t)=v_{1-2}(t)=\frac{t}{t+1}=v_{A-B}(t)
$$

Example 2.18 Let $X=\mathbb{R}^{2}, A=\{(x, 0): 0 \leq x \leq 1\}, B_{1}=\{(x, y): x+y=1,-1 \leq x \leq 0\}, B_{2}=$ $\{(x, 1): 0 \leq x \leq 1\}, B=B_{1} \cup B_{2}$, and $v_{\left(x, x^{\prime}\right)}(t)=\frac{t}{t+|x|+\left|x^{\prime}\right|}$. It is easy to see that $\left(X, v, \Delta_{m}\right)$ is a probabilistic Banach space, $v_{A-B}(t)=\frac{t}{t+1}, B$ is not convex but is a $(0,1)$-star-shaped set, and $A$ is $(0,0)$-star-shaped set. Clearly, $A_{0}=A$ and $B_{0}=B_{2}$. So

$$
v_{(0,0)-(0,1)}(t)=\frac{t}{t+|0|+|1|}=\frac{t}{t+1}=v_{A-B}(t)
$$

and $(A, B)$ is a semisharp proximinal pair. Suppose that $T: A \rightarrow B$ is defined by

$$
T(x, 0)= \begin{cases}(0,1), & x=0 \\ (\sin x, 1), & x \neq 0\end{cases}
$$

and $(u, 0),(v, 0),(x, 0),(y, 0) \in A$ are such that

$$
v_{(u, 0)-T(x, 0)}(t)=v_{A-B}(t)=\frac{t}{t+1}=v_{(v, 0)-T(y, 0)}(t) .
$$


If $x=y=0$, then $u=v=0$, and therefore

$$
v_{(u, 0)-(v, 0)}(t)=v_{(0,0)-(0,0)}(t)=1=v_{(x, 0)-(y, 0)}(t) .
$$

If $x, y \neq 0$, then $u=\sin x, v=\sin y$, and therefore

$$
\begin{aligned}
v_{(u, 0)-(v, 0)}(t) & =v_{(\sin x, 0)-(\sin y, 0)}(t)=\frac{t}{t+|\sin x-\sin y|} \\
& \geq \frac{t}{t+|x-y|} \\
& =v_{(x, 0)-(y, 0)}(t) .
\end{aligned}
$$

If $x=0$ and $y \neq 0$, then $u=0$ and $v=\sin y$, and therefore

$$
v_{(u, 0)-(v, 0)}(t)=v_{(0,0)-(\sin y, 0)}(t)=\frac{t}{t+|\sin y|} \geq \frac{t}{t+|y|} \geq v_{(0,0)-(y, 0)}(t) .
$$

If $x \neq 0$ and $y=0$, then $u=\sin x$ and $v=0$, and therefore

$$
v_{(u, 0)-(v, 0)}(t)=v_{(\sin x, 0)-(0,0)}(t)=\frac{t}{t+|\sin x|} \geq \frac{t}{t+|x|} \geq v_{(x, 0)-(0,0)}(t)
$$

Hence, $T$ is proximal nonexpansive, and $T\left(A_{0}\right) \subseteq B_{2}=B_{0}$, so all the hypotheses of Theorem 2.7 are satisfied, and we also have

$$
v_{(0,0)-T(0,0)}(t)=v_{(0,0)-(0,1)}(t)=\frac{t}{t+1}=v_{A-B}(t)
$$

Example 2.19 Let $X=\mathbb{R}, A=[0,1], B=\left[\frac{15}{8}, 2\right]$, and $v_{x}(t)=\frac{t}{t+|x|}$. Clearly, $\left(X, v, \Delta_{m}\right)$ is a probabilistic Banach space, $v_{A-B}(t)=\frac{t}{t+\frac{7}{8}}$, the pair $(A, B)$ has the $P$-property, $A_{0}=\{1\}$, and $B_{0}=\left\{\frac{15}{8}\right\}$. If $T x=-\frac{1}{8} x+2$, then $T\left(A_{0}\right)=\{T(1)\}=\left\{\frac{15}{8}\right\}=B_{0}$. Let $x, y \in A$. Then we have

$$
v_{T x-T y}(t)=v_{-\frac{1}{8}(x-y)}(t)=v_{x-y}(8 t) \geq v_{x-y}(t)
$$

Therefore, all the hypotheses of Corollary 2.12 are satisfied, and hence $T$ has a best proximity point, and we also have

$$
v_{1-T 1}(t)=v_{1-\frac{15}{8}}(t)=\frac{t}{t+\frac{7}{8}}=v_{A-B}(t)
$$

\section{Competing interests}

The authors declare that they have no competing interests.

\section{Authors' contributions}

All authors have contributed in obtaining the new results presented in this article. All authors read and approved the final manuscript.

\section{Acknowledgements}

The authors would like to express their sincere appreciation to the Shahrekord University and the Center of Excellence for Mathematics for financial support.

Received: 5 July 2015 Accepted: 11 December 2015 Published online: 15 January 2016 


\section{References}

1. Fan, K: Extensions of two fixed point theorems of F.E. Browder. Math. Z. 122, 234-240 (1969)

2. Reich, S: Approximate selections, best approximations, fixed points, and invariant sets. J. Math. Anal. Appl. 62(1), 104-113 (1978)

3. Reich, S: Fixed points in locally convex spaces. Math. Z. 125, 17-31 (1972)

4. Sehgal, VM, Singh, SP: A generalization to multifunctions of Fan's best approximation theorem. Proc. Am. Math. Soc. 102(3), 534-537 (1988)

5. Eldred, AA, Kirk, WA, Veeramani, P: Proximinal normal structure and relatively nonexpansive mappings. Stud. Math. 171(3), 283-293 (2005)

6. Al-Thagafi, MA, Shahzad, N: Best proximity pairs and equilibrium pairs for Kakutani multimaps. Nonlinear Anal. 70(3), 1209-1216 (2009)

7. Al-Thagafi, MA, Shahzad, N: Best proximity sets and equilibrium pairs for a finite family of multimaps. Fixed Point Theory Appl. 2008, Article ID 457069 (2008)

8. Al-Thagafi, MA, Shahzad, N: Convergence and existence results for best proximity points. Nonlinear Anal. 70(10), 3665-3671 (2009)

9. Eldred, AA, Veeramani, P: Existence and convergence of best proximity points. J. Math. Anal. Appl. 323, 1001-1006 (2006)

10. Di Bari, C, Suzuki, T, Vetro, C: Best proximity points for cyclic Meir-Keeler contractions. Nonlinear Anal. 69(11), 3790-3794 (2008)

11. Karpagam, S, Agrawal, S: Best proximity point theorems for $p$-cyclic Meir-Keeler contractions. Fixed Point Theory Appl. 2009, Article ID 197308 (2009)

12. Kim, WK, Kum, S, Lee, KH: On general best proximity pairs and equilibrium pairs in free abstract economies. Nonlinear Anal. 68(8), 222-2216 (2008)

13. Kirk, WA, Reich, S, Veeramani, P: Proximinal retracts and best proximity pair theorems. Numer. Funct. Anal. Optim. 24, 851-862 (2003)

14. Mongkolkeha, C, Kumam, P: Best proximity point theorems for generalized cyclic contractions in ordered metric spaces. J. Optim. Theory Appl. (2012). doi:10.1007/s10957-012-9991-y

15. Mongkolkeha, C, Kumam, P: Some common best proximity points for proximity commuting mappings. Optim. Lett (2012). doi:10.1007/s11590-012-0525-1

16. Prolla, JB: Fixed-point theorems for set-valued mappings and existence of best approximants. Numer. Funct. Anal. Optim. 5(4), 449-455 (1983)

17. Sadiq Basha, S, Veeramani, P: Best approximations and best proximity pairs. Acta Sci. Math. (Szeged) $63,289-300$ (1997)

18. Sadiq Basha, S, Veeramani, P: Best proximity pair theorems for multifunctions with open fibres. J. Approx. Theory 103, 119-129 (2000)

19. Sadiq Basha, S, Veeramani, P, Pai, DV: Best proximity pair theorems. Indian J. Pure Appl. Math. 32, 1237-1246 (2001)

20. Sehgal, VM, Singh, SP: A theorem on best approximations. Numer. Funct. Anal. Optim. 10(1-2), 181-184 (1989)

21. Sintunavarat, W, Kumam, P: Coupled best proximity point theorem in metric spaces. Fixed Point Theory Appl. 2012 93 (2012). doi:10.1186/1687-1812-2012-93

22. Srinivasan, PS: Best proximity pair theorems. Acta Sci. Math. (Szeged) 67, 421-429 (2001)

23. Wlodarczyk, K, Plebaniak, R, Banach, A: Best proximity points for cyclic and noncyclic set-valued relatively quasi-asymptotic contractions in uniform spaces. Nonlinear Anal. 70(9), 3332-3341 (2009)

24. Wlodarczyk, K, Plebaniak, R, Banach, A: Erratum to: 'Best proximity points for cyclic and noncyclic set-valued relatively quasi-asymptotic contractions in uniform spaces'. Nonlinear Anal. 71, 3583-3586 (2009)

25. Wlodarczyk, K, Plebaniak, R, Obczynski, C: Convergence theorems, best approximation and best proximity for set-valued dynamic systems of relatively quasi-asymptotic contractions in cone uniform spaces. Nonlinear Anal. 72 794-805 (2010)

26. Menger, K: Statistical metrics. Proc. Natl. Acad. Sci. USA 28, 535-537 (1942)

27. Schweizer, B, Sklar, A: Probabilistic Metric Spaces. North-Holland Series in Probability and Applied Mathematics. North-Holland, New York (1983)

28. Schweizer, B, Sklar, A: Statistical metric spaces. Pac. J. Math. 10, 313-334 (1960)

29. Chang, SS, Cho, YJ, Kang, SM: Nonlinear Operator Theory in Probabilistic Metric Spaces. Nova Science Publishers, New York (2001)

30. Chang, SS, Lee, BS, Cho, YJ, Chen, YQ, Kang, SM, Jung, JM: Generalized contraction mapping principle and differential equations in probabilistic metric spaces. Proc. Am. Math. Soc. 124, 2367-2376 (1996)

31. El Naschie, MS: Fuzzy dodecahedron topology and E-infinity spacetimes as a model for quantum physics. Chaos Solitons Fractals 30(5), 1025-1033 (2006)

32. El Naschie, MS: On gauge invariance, dissipative quantum mechanics and self-adjoint sets. Chaos Solitons Fractals 32(2), 271-273 (2007)

33. El Naschie, MS: P-Adic analysis and the transfinite E8 exceptional Lie symmetry group unification. Chaos Solitons Fractals 38(3), 612-614 (2008)

34. Sehgal, VM, Bharucha-Reid, AT: Fixed points of contraction mappings on probabilistic metric spaces. Math. Syst. Theory 6, 97-102 (1972)

35. Jungck, G: Compatible mappings and common fixed points. Int. J. Math. Math. Sci. 9, 771-779 (1986)

36. O'Regan, D, Saadati, R: Nonlinear contraction theorems in probabilistic spaces. Appl. Math. Comput. 195, 86-93 (2008)

37. Razani, A, Shirdaryazdi, M: A common fixed point theorem of compatible maps in Menger space. Chaos Solitons Fractals 32, 26-34 (2007)

38. Su, Y, Zhang, J: Fixed point and best proximity point theorems for contractions in new class of probabilistic metric spaces. Fixed Point Theory Appl. 2014,170 (2014)

39. Hadžić, O, Pap, E: Fixed Point Theory in Probabilistic Metric Spaces. Kluwer Academic, Dordrecht (2001) 